\title{
SOBRE LA FECHA DE HIPP. DE GLANDULIS
}

IgnaCio Rodriguez Alfageme

Universidad Complutense

1. El tratado De glandulis ha planteado un problema de datación y autoría desde que $\mathrm{H}$. Diels en 1910, criticando la tesis de Ermerins, que aceptaba la unidad de autor para este tratado y De articulis, lo consideró una falsificación de época alejandrina. En la última edición del tratado R. Joly ${ }^{1}$, pasando revista a las conclusiones a las que había llegado H. Mørland ${ }^{2}$ en 1941 sobre su lengua, lo fecha a finales del siglo $\mathrm{V}$ o principios del IV a.C. Pero la reseña de A. Anastassiou $^{3}$ a la edición de $R$. Joly ha vuelto a plantear el problema de la fecha y la autoría de este tratado. En líneas generales este autor considera que la hipótesis de que es una imitación de los tratados quirúrgicos, está bien fundada, atendiendo en parte a los argumentos de Diels y a los de Morland (presencia de arcaísmos áticos y jónicos sin discriminación, etc.), y añadiendo de su parte el uso del verbo év $\alpha \pi \varepsilon^{\prime} \theta \varepsilon \tau o$, propio de los prosistas de época imperial y de Galeno en uso técnico, y el empleo de $\sigma \varphi e^{\prime} \tau \varepsilon \rho \varsigma \zeta$ como reflexivo posesivo de tercera persona, ajeno a la prosa hasta época tardía, aunque conocido de la poesía antigua.

2. La crítica que se puede hacer a todos estos argumentos es semejante: todos ellos se basan en la presencia de una construcción o de un término preciso

1 Hippocrate. Tome XIII, París 1978.

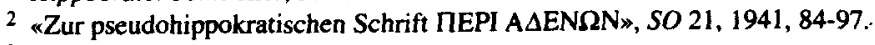

3 Gnomon 52, 1980, 306-8. 
que sólo se atestigua en cierta época. Pero es difícil demostrar, para palabras que tienen una frecuencia baja, que un uso específico, en el caso del vocabulario, no haya existido en época anterior a la que dan nuestros diccionarios, con lo que siempre queda un margen de duda sobre la corrección de las conclusiones a las que han llegado unos y otros. El estudio de las coincidencias de léxico puede ser muy fructífero, pero difícilmente por sí sólo puede servir para fechar un tratado. Para fijar una fecha hay que recurrir a fenómenos linguísticos que reflejen la evolución de la lengua. Con ese fin hemos estudiado la frecuencia de las conjunciones y partículas, porque al ser muy alta en cualquier estado de lengua tiende a ser constante para cada época ${ }^{4}$. Si realmente De glandulis es una imitación, como se suele suponer, no es fácil que el autor logre deshacerse de sus esquemas lingüísticos para adoptar los de su modelo, porque no se trata ya de imitar una frase o una forma arcaica, sino de la frecuencia con que se utiliza una conjunción o una partícula, de modo que se podría decir que la frecuencia traiciona al imitador.

3. Aceptando esta hipótesis de trabajo he estudiado las distintas partículas que sirven para unir las oraciones del De glandulis con el fin de intentar dar una imagen más precisa de sus afinidades lingüísticas. Los datos estadísticos no tienen un peso decisivo, puesto que lo único que nos dicen es lo que no es el texto, pero pueden servir para encaminar la investigación por ciertos derroteros con exclusión de otros que en principio podrían ser igualmente probables.

4. En un primer momento se trata de comprobar las partículas conectivas de los distintos tratados con los que muestra mayor afinidad De glandulis, es decir: De articulis y De locis in homine, y demostrar, si es posible, que De glandulis no es obra del mismo autor que cualquiera de estos tratados. Por otra parte, hay que ver los indicios con que podemos contar para asignar una fecha más aproximada al tratado, cifrándola entre la doble posibilidad de que sea una obra del siglo V-IV, o bien una obra de época posterior. Con ese fin he recogido todas las partículas que sirven para introducir oraciones en el De glandulis y las he confrontado con una muestra que comprende las primeras $\mathbf{2 8 0}$ oraciones de los dos tratados que nos sirven de término de comparación. He procedido así en lugar de recurrir a una muestra aleatoria porque desde la publicación de la concordancia de Maloney tenemos a nuestra disposición los datos totales para

\footnotetext{
${ }^{4}$ Cf. G. Herdan, Quantitative linguistics, London 1969, pp. 77 s.
} 
cada uno de los tratados del $\mathrm{CH}$. Pero tanto esta concordancia como el léxico del $\mathrm{CH}^{5}$ no recogen las frecuencias de las dos partículas fundamentales, kơ y $\delta \varepsilon$, aunque se nos dice que, junto con el artículo, ocuparían unas 85.000 líneas, lo que equivale a decir que aparecen ese número de veces ${ }^{6}$, pero no se distinguen las frecuencias respectivas ni es posible determinar a priori la frecuencia de empleo de k $\alpha \boldsymbol{~ y ~} \delta \varepsilon$ para unir oraciones, para unir palabras dentro de una oración y en uso adverbial. Y estos datos no pueden pasarse por alto para intentar vislumbrar una fecha.

5. Las partículas son las siguientes:

Clasificación de las conectivas por orden de frecuencia en De Glandulis

\begin{tabular}{|c|c|c|}
\hline Part & Con & $G l$ \\
\hline$\kappa \alpha t$ & - & 77 \\
\hline$\delta \varepsilon$ & - & 55 \\
\hline asín. & - & 30 \\
\hline yap & 22 & 19 \\
\hline$n v$ & 16 & 16 \\
\hline$\alpha \lambda \lambda \alpha$ & 10 & 10 \\
\hline$\mu \dot{k}^{\prime}$ & 19 & 8 \\
\hline ơं $\tau \omega$ & 8 & 4 \\
\hline$\varepsilon^{\prime}$ & 7 & 7 \\
\hline$\tau \varepsilon \operatorname{cod}$ & - & 7 \\
\hline 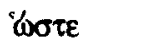 & 6 & 6 \\
\hline "botls & 5 & 5 \\
\hline '́́mov & 4 & 4 \\
\hline
\end{tabular}

5 G. Malloney Y W. Frohn, Concordantia in Corpus Hippocraticum, Hildesheim-ZürichNew York, 1986; KÜHN-FLEISCHER, Index hippocraticus, Göttigen 1989.

${ }^{6}$ Haciendo los cálculos más exactos el número de veces que aparecen estas tres palabras debe estar próximo a 87.426 resultante de multiplicar por 60 (número de líneas por página) por 4.896 (número de páginas) restándole el número de lemas, 11.752, y comparándolo todo ello con el total de palabras que da Malloney (367.814) para el $C H$; cf. «L'emploi des particules dans les oeuvres d'Hippocrate», RELO 1980, 4, pp. 1-31. 
Clasificación de las conectivas por orden de frecuencia en De Glandulis (Continuación)

\begin{tabular}{|c|c|c|}
\hline Part & Con & $G l$ \\
\hline 'ó & 4 & 4 \\
\hline 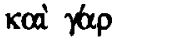 & - & 3 \\
\hline$\varepsilon \pi \eta$ & 3 & 3 \\
\hline$\omega \varsigma$ & 2 & 2 \\
\hline$\tau \varepsilon$ & 13 & 2 \\
\hline 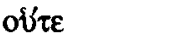 & 5 & 2 \\
\hline$\varepsilon \tilde{\varepsilon} \sigma \tau^{\prime \prime} \not \dot{\alpha} v$ & 2 & 2 \\
\hline$\omega \sigma \pi \varepsilon \rho$ & 2 & 2 \\
\hline$\delta \pi \omega_{\varsigma} \pi \varepsilon \rho$ & 2 & 2 \\
\hline exel & 1 & 1 \\
\hline ช่นย & 2 & 1 \\
\hline 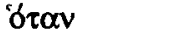 & 1 & 1 \\
\hline$\delta \tilde{\delta} \delta \tau \tilde{\tau}$ & 1 & 1 \\
\hline$\delta \tilde{\delta} \delta \sigma 0 \varsigma$ & 2 & 1 \\
\hline ofor & 5 & 1 \\
\hline$\mu \dot{\varepsilon} v \not \not \alpha \rho$ & - & 1 \\
\hline$\kappa \alpha \tau \alpha \tau \alpha u \alpha \alpha$ & - & 1 \\
\hline кoa' $\delta$ h & - & 1 \\
\hline$\mu \eta$ & 4 & 1 \\
\hline
\end{tabular}

La primera columna recoge las cifras absolutas, tal como aparecen en la Concordancia del $\mathrm{CH}$ y la segunda nuestros datos, que son el resultado de haber recogido las partículas que funcionan de hecho para introducir oraciones en el texto editado por $\mathbf{R}$. Joly. De ahí las diferencias en las cifras y la ausencia de palabras tales como $k \alpha \alpha$ y $\delta \varepsilon$, o la falta de datos sobre el asíndeton y algunas otras combinaciones de partículas. Aparte de estos datos, en el texto que nos ocupa aparece un par de veces la partícula $\delta \eta(7,3)$, una de ellas recogida en

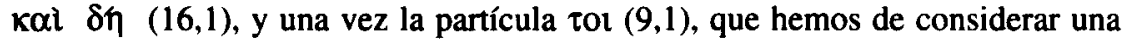
verdadera rareza ${ }^{7}$.

7 Sólo aparece, aparte de este tratado, en De prisca medicina, De victu in acutis, Acut(sp), De articulis, De morb. II, Prorh., en la carta de Demócrito a Hipócrates y en el Discurso de Tésalo, todos ellos atestiguan también la partícula $\delta\lceil$. Su rareza invita a eliminarla leyendo $\tau \varepsilon$, de forma que tendriamos un doble atributo de ' $\alpha \delta \varepsilon v \varepsilon \zeta$ unido por $\tau \varepsilon$ koa'. Hay un paralelo en el doble atributo que

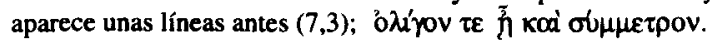


6. Pero estas cifras totales no dan idea de como se reparten las partículas en el texto. Para poder apreciar la regularidad de ese reparto, lo he dividido en cinco grupos de 56 oraciones. El cuadro siguiente recoge el resultado de este cómputo, hecho a partir del texto de R. Joly:

Distribución de las partículas en De gland ${ }^{8}$

\begin{tabular}{|c|c|c|c|c|c|c|c|c|c|}
\hline Part & $A$ & $B$ & $C$ & $D$ & $E$ & Tot. & $\bar{x}$ & $\sigma 2$ & $\sigma$ \\
\hline asin. & 4 & 6 & 8 & 7 & 5 & 30 & 6.00 & 2.00 & 1.41 \\
\hline$\alpha \lambda \lambda \alpha \alpha$ & 2 & 4 & 2 & 1 & 1 & 10 & 2.00 & 1.20 & 1.10 \\
\hline$\S \notin$ & 10 & 13 & 11 & 13 & 8 & 55 & 11.00 & 3.60 & 1.90 \\
\hline$\varepsilon i$ & 2 & 0 & 1 & 4 & 0 & 7 & 1.40 & 2.24 & 1.50 \\
\hline éced & 0 & 0 & 1 & 0 & 0 & 1 & 0.20 & 0,16 & 0,40 \\
\hline$\varepsilon \pi$ हhV & 2 & 1 & 0 & 0 & 0 & 3 & 0.60 & 0.64 & 0.80 \\
\hline 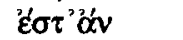 & 0 & 1 & 0 & 1 & 0 & 2 & 0.40 & 0.24 & 0.49 \\
\hline xap & 3 & 5 & 5 & 2 & 4 & 19 & 3.80 & 1.36 & 1.17 \\
\hline nv & 2 & 1 & 5 & 5 & 3 & 16 & 3.20 & 2.56 & 1.60 \\
\hline кo' of & 0 & 0 & 0 & 0 & 1 & 1 & 0.20 & 0.16 & 0.40 \\
\hline кoa' $\gamma \alpha \rho$ & 0 & 3 & 0 & 0 & 0 & 3 & 0.60 & 1.44 & 1.20 \\
\hline$\kappa \propto \alpha$ & 13 & 11 & 15 & 17 & 21 & 77 & 15.40 & 11.84 & 3.44 \\
\hline$\kappa \alpha \tau \alpha \tau \alpha v \alpha$ & 0 & 0 & 1 & 0 & 0 & 1 & 0.20 & 0.16 & 0.40 \\
\hline$\mu \dot{E v}$ & 4 & 1 & 1 & 0 & 2 & 8 & 1.60 & 1.84 & 1.36 \\
\hline$\mu \varepsilon_{v} \gamma^{\prime} \alpha \rho \rho$ & 0 & 0 & 0 & 1 & 0 & 1 & 0.20 & 0.16 & 0.40 \\
\hline of́os & 0 & 1 & 0 & 0 & 0 & 1 & 0.20 & 0.16 & 0.40 \\
\hline 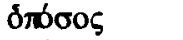 & 0 & 1 & 0 & 0 & 0 & 1 & 0.20 & 0.16 & 0.40 \\
\hline$\delta \pi \delta \tau \tau \alpha$ & 0 & 0 & 1 & 0 & 0 & 1 & 0.20 & 0.16 & 0.40 \\
\hline 'ómov & 3 & 1 & 0 & 0 & 0 & 4 & 0.80 & 1.36 & 1.17 \\
\hline 'ó $\pi \omega s$ & 0 & 0 & 2 & 0 & 0 & 2 & 0.40 & 0.64 & 0.80 \\
\hline ós & 1 & 1 & 0 & 0 & 2 & 4 & 0.80 & 0.56 & 0.75 \\
\hline 'óons & 2 & 1 & 0 & 0 & 2 & 5 & 1.00 & 0.80 & 0.89 \\
\hline$\delta \tau \alpha \alpha v$ & 0 & 0 & 1 & 0 & 0 & 1 & 0.20 & 0.16 & 0.40 \\
\hline ơ์ $\varepsilon \varepsilon$ & 1 & 1 & 0 & 0 & 0 & 2 & 0.40 & 0.24 & 0.49 \\
\hline
\end{tabular}

8 Los cálculos estadísticos que se ofrecen en el cuadro sólo son significativos para las partículas con una frecuencia alta. 
Distribución de las partículas en $D e$ gland ${ }^{8}$

(Continuación)

\begin{tabular}{|c|c|c|c|c|c|c|c|c|c|}
\hline Part & $A$ & $B$ & $C$ & $D$ & $E$ & Tot. & $\bar{x}$ & $\sigma 2$ & $\sigma$ \\
\hline 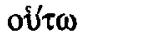 & 2 & 0 & 0 & 1 & 1 & 4 & 0.80 & 0.56 & 0.75 \\
\hline$\tau \varepsilon$ & 0 & 1 & 1 & 0 & 0 & 2 & 0.40 & 0.24 & 0.49 \\
\hline$\tau \varepsilon \mathrm{K \alpha}$ & 0 & 1 & 1 & 4 & 1 & 7 & 1.40 & 1.84 & 1.36 \\
\hline$\tau \sigma \tau \varepsilon$ & 0 & 1 & 0 & 0 & 0 & 1 & 0.20 & 0.16 & 0.40 \\
\hline$\omega s$ & 2 & 0 & 0 & 0 & 0 & 2 & 0.40 & 0.64 & 0.80 \\
\hline$\omega \sigma \pi \varepsilon \rho$ & 0 & 0 & 0 & 0 & 2 & 2 & 0.40 & 0.64 & 0.80 \\
\hline 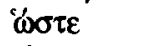 & 3 & 1 & 0 & 0 & 2 & 6 & 1.20 & 1.36 & 1.17 \\
\hline$\mu \eta \tilde{\eta}$ & 0 & 0 & 0 & 0 & 1 & 1 & 0.20 & 0.16 & 0.40 \\
\hline
\end{tabular}

Se nota que las partículas raras tienden a acumularse en el mismo sector o en sectores contiguos, como si su aparición en un momento dado aumentara sus probabilidades de repetición. Sin duda este cuadro puede ser muy significativo para determinar y comparar los rasgos de estilo del tratado, pero no podemos ocuparnos aquí de este asunto.

7. El modo más sencillo de comprobar que se trata de autores diferentes en los tres tratados afines es comparar estas obras con De glandulis mediante el uso de algún test estadístico; en este caso un test de Pearson puede bastar ${ }^{9}$, pero también podemos utilizar con el mismo fin un test de desviaciones reducidas, ya que conocemos la frecuencia absoluta de las partículas gracias a la concordancia de Maloney. Los resultados de la aplicación de estos tests pueden verse en las tablas siguientes:

${ }^{9}$ Para el significado de estos procedimientos estadísticos, vid. CH. Muller, Estadistica Lingüistica, Madrid 1968. 
Comparación entre De loc in hom. y De gland

\begin{tabular}{|c|c|c|c|c|c|c|c|c|c|}
\hline Part & Tot & $L C$ & $t$ & $d$ & $\chi^{2}$ & $G l$ & $t$ & $d$ & $x^{2}$ \\
\hline$\gamma \alpha \alpha \rho$ & 86 & 64 & 74 & -10 & 1.35 & 22 & 12 & 10 & 8.33 \\
\hline$k v$ & 119 & 103 & 103 & 0 & 0.00 & 16 & 16 & 0 & 0.00 \\
\hline$\mu \dot{\varepsilon} v$ & 117 & 98 & 101 & -3 & 0.09 & 19 & 16 & 3 & 0.56 \\
\hline "os & 56 & 52 & 48 & 4 & 0.33 & 4 & 8 & -4 & 2.00 \\
\hline$\tau \varepsilon$ & 67 & 54 & 58 & -4 & 0.28 & 13 & 9 & 4 & 1.78 \\
\hline$\delta \tau \alpha \alpha$ & 51 & 50 & 44 & 6 & 0.82 & 1 & 7 & -6 & 5.14 \\
\hline$\mu 斤$ & 84 & 80 & 73 & 7 & 0.67 & 4 & 11 & -7 & 4.45 \\
\hline
\end{tabular}

$\chi^{2}=25,8 \quad 6$ gdl $0,001=22,457$

A pesar de que sólo hemos podido recoger en este cuadro las partículas más frecuentes que registra la concordancia de Maloney, el valor de $\chi^{2}=25,8$ con 6 gdl. sobrepasa el límite del 0,001; podemos, pues, afirmar con seguridad que se trata de obras de autores distintos.

8. Este resultado nos sirve de piedra de toque para la comparación de este tratado con De articulis. Los resultados ofrecen un aspecto muy distinto:

Comparación entre De art. y De gland. $\chi^{2}$

\begin{tabular}{|c|c|c|c|c|c|c|c|c|c|}
\hline Part & Tot & $A n$ & $t$ & $d$ & $\chi^{2}$ & $G l$ & $t$ & $d$ & $\chi^{2}$ \\
\hline$\alpha \lambda \lambda \alpha \alpha$ & 117 & 107 & 100 & -3 & 0.08 & 10 & 7 & 3 & 1.29 \\
\hline$\gamma \alpha p$ & 286 & 264 & 269 & -5 & 0.09 & 22 & 17 & 5 & 1.47 \\
\hline$\eta v$ & 213 & 197 & 200 & -3 & 0.04 & 16 & 13 & 3 & 0.69 \\
\hline$\mu \hat{\varepsilon} v$ & 385 & 366 & 362 & 4 & 0.04 & 19 & 23 & -4 & 0.70 \\
\hline 'os & 144 & 140 & 135 & 5 & 0.19 & 4 & 9 & -5 & 2.78 \\
\hline$\tau \varepsilon$ & 167 & 154 & 157 & -3 & 0.06 & 13 & 10 & 3 & 0.90 \\
\hline$\mu \eta \dot{n}$ & 175 & 171 & 165 & 6 & 0.22 & 4 & 10 & -6 & 3.60 \\
\hline
\end{tabular}

$\chi^{2}=12,5 \quad 6 \operatorname{gdl} 0,05=12,592$

$\mathrm{t}=$ teórica

$\mathrm{d}=$ diferencia entre la cantidad observada y la teorica $« t »$. 
El valor de $\chi^{2}$ ha descendido mucho: 12,5 para 6 gdl. está cercano al 0,05 ; es decir, en más de un $5 \%$ de los casos nos encontramos con una distribución aleatoria. Es, por lo tanto, difícil que ambas obras pertenezcan al mismo autor, pero no puede excluirse esa posibilidad. Sin embargo, hay un problema estadístico, ya que los efectivos teóricos son bajos en el caso de ỏ $\alpha \lambda \lambda \alpha ́ \alpha$ y 'ós. Para evitar el inconveniente que esto supone (las cifras teóricas bajas falsean los resultados), los he eliminado para rehacer de nuevo los cálculos. El cuadro ofrece ahora el siguiente aspecto:

Comparación entre De art. y De gland. $\chi^{2}$

\begin{tabular}{|c|c|c|c|c|c|c|c|c|c|}
\hline Part & Tot & $A r t$ & $t$ & $d$ & $x^{2}$ & $G l$ & $t$ & $d$ & $\chi^{2}$ \\
\hline$\gamma \alpha p$ & 286 & 264 & 269 & -5 & 0.09 & 22 & 17 & 5 & 1.47 \\
\hline inv & 213 & 197 & 200 & -3 & 0.04 & 16 & 13 & 3 & 0.69 \\
\hline$\mu \dot{\varepsilon} \nu$ & 385 & 366 & 362 & 4 & 0.04 & 19 & 23 & -4 & 0.70 \\
\hline$\tau \varepsilon$ & 167 & 154 & 157 & -3 & 0.06 & 13 & 10 & 3 & 0.90 \\
\hline$\mu \eta$ & 175 & 171 & 165 & 6 & 0.30 & 4 & 11 & -7 & 4.45 \\
\hline
\end{tabular}

Ahora $\chi^{2}=8,74$ con 4 gdl., lo que está próximo al valor que da la tabla para el percentil del 0,05: 9,488. Podemos, pues, decir que en más de un 5\% de los casos esta distribución se debe al azar. Para que este resultado sea posible es necesario que la distribución de las partículas tienda a ser aleatoria. No puede excluirse que los dos tratados sean del mismo autor por este procedimiento.

9. Parece, por lo tanto, oportuno comprobar el resultado con otro test estadístico. Como los datos de que disponemos no permiten una comparación de los efectivos totales, es preciso recurrir a la comparación de medias en corpora análogos: por ello he tomado los datos que aparecen en el cuadro del $\S 6$ para compararlos con muestras iguales tomadas del principio de Sobre las articulaciones. Los resultados se ofrecen en los cuadros siguientes: 
Distribución de conectivas en 5 muestras de 56 oraciones De articulis

\begin{tabular}{|l|r|r|r|r|r|r|r|r|r|}
\hline \multicolumn{1}{|c|}{ Part } & \multicolumn{1}{c|}{$A$} & \multicolumn{1}{c|}{$B$} & \multicolumn{1}{c|}{$C$} & \multicolumn{1}{c|}{$D$} & $E$ & Tot. & $\bar{x}$ & $\sigma 2$ & $\sigma$ \\
\hline asín. & 1 & 2 & 2 & 1 & 4 & 10 & 2.00 & 1.20 & 1.10 \\
$\varnothing \varepsilon$ & 14 & 24 & 14 & 14 & 12 & 78 & 15.60 & 18.24 & 4.27 \\
Kơt & 5 & 4 & 5 & 4 & 9 & 27 & 5.40 & 3.44 & 1.85 \\
xap & 4 & 4 & 3 & 4 & 6 & 21 & 4.20 & 0.96 & 0.98 \\
cond & 6 & 3 & 4 & 3 & 1 & 17 & 3.40 & 2.64 & 1.62 \\
ocoor & 13 & 7 & 12 & 18 & 10 & 60 & 12.00 & 13.20 & 3.63 \\
orel & 7 & 3 & 1 & 2 & 6 & 19 & 3.80 & 5.36 & 2.32 \\
osub & 6 & 9 & 15 & 10 & 7 & 47 & 9.40 & 9.84 & 3.14 \\
\hline
\end{tabular}

cond $=\varepsilon i$, inv

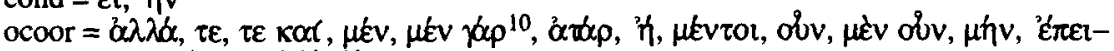

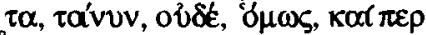

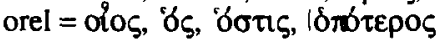

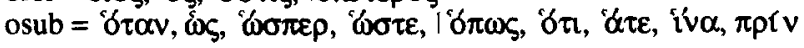

\section{De glandulis}

\begin{tabular}{|l|r|r|r|r|r|r|r|r|r|}
\hline \multicolumn{1}{|c|}{ Part } & $A$ & \multicolumn{1}{c|}{$B$} & $C$ & $D$ & $E$ & Tot. & $\tilde{x}$ & $\sigma 2$ & $\sigma$ \\
\hline asín. & 6 & 7 & 9 & 8 & 6 & 36 & 7.20 & 1.36 & 1.17 \\
¿£ & 10 & 13 & 11 & 13 & 8 & 55 & 11.00 & 3.60 & 1.90 \\
Kợ & 13 & 13 & 15 & 17 & 22 & 80 & 16.00 & 11.20 & 3.35 \\
xap & 3 & 5 & 5 & 2 & 4 & 19 & 3.80 & 1.36 & 1.17 \\
cond & 4 & 1 & 6 & 9 & 3 & 23 & 4.60 & 7.44 & 2.73 \\
ocoor & 7 & 8 & 5 & 6 & 4 & 30 & 6.00 & 2.00 & 1.41 \\
orel & 6 & 5 & 0 & 0 & 4 & 15 & 3.00 & 6.40 & 2.53 \\
osub & 7 & 4 & 5 & 1 & 5 & 22 & 4.40 & 3.84 & 1.96 \\
\hline
\end{tabular}

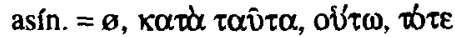

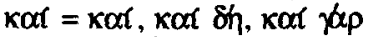

cond $=\varepsilon i$, inv

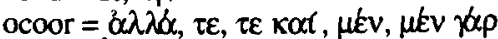

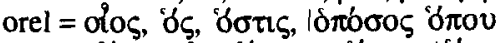

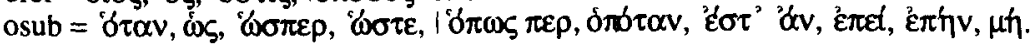

10 La barra vertical separa las partículas que no aparecen en el otro tratado. 
Aun sin fijarnos en las cifras resalta a primera vista la riqueza de medios de coordinación del primero de los tratados frente a De glandulis.

10. Para poder comparar mejor estos resultados se ha procedido a comparar las medidas mediante el test de Student-Fisher ${ }^{11}$, los resultados no dejan lugar a dudas de que se trata de autores distintos:

\section{Aplicación del test de Student a De art y Gland}

\begin{tabular}{|c|c|c|c|c|c|c|c|}
\hline Pant & $A r$ & $\bar{x}$ & $\sigma^{2}$ & $G l$ & $\bar{x}$ & $\sigma^{2}$ & $t$ \\
\hline asín. & 10 & 2.00 & 1.20 & 36 & 7.20 & 1.36 & 5.30 \\
\hline$\delta \varepsilon$ & 78 & 15.60 & 18.24 & 55 & 11.00 & 3.60 & 1.97 \\
\hline$\kappa \propto \alpha t$ & 27 & 5.40 & 3.44 & 80 & 16.00 & 11.20 & 5.54 \\
\hline$x_{\alpha}^{\prime} \alpha$ & 21 & 4.20 & 0.96 & 19 & 3.80 & 1.36 & 0.49 \\
\hline cond & 17 & 3.40 & 2.64 & 23 & 4.60 & 7.44 & 0.76 \\
\hline ocoor & 60 & 12.00 & 13.20 & 30 & 6.00 & 2.00 & 3.08 \\
\hline orel & 19 & 3.80 & 5.36 & 15 & 3.00 & 6.40 & 0.47 \\
\hline osub & 47 & 9.40 & 9.84 & 22 & 4.40 & 3.84 & 2.70 \\
\hline
\end{tabular}

Con $8 \mathrm{gdl}$. el umbral del 0.05 da un valor para «t» $=2.306$, superado por el asíndeton, $k \alpha \chi$, las oraciones coordinadas y las subordinadas; además $\delta \varepsilon$ supera el 0.10 . En resumidas cuentas sólo las medias de las oraciones de relativo, las condicionales y las introducidas por үáp son comparables. Aún descontando que las coordinadas tienen una desviación típica que hace difícil la comparación de las medias, hemos de concluir que se trata de dos autores distintos. Además, aunque el reparto de las oraciones condicionales y las oraciones de relativo sea semejante, se trata de realidades muy distintas. En el caso de las condicionales las diferencias de cifras se deben exclusivamente a $\eta \vee$ que aparece 16 veces en De glandulis.

1 Este test ha sido aplicado para resolver el problema de atribución de autor de las dos partes del Roman de Tristán por M. DEBIEVRE, «Essai d'appliccation des méthodes de la statistique linguistique au problème posé par l'attribution du texte de la version française du Roman de Tristan", en J. DAvid \& R. MARTIN, Etudes de statistique linguistique, Metz 1977, pp. 35-54. 
11. Más interesante aún es el caso de las oraciones de relativo. En De

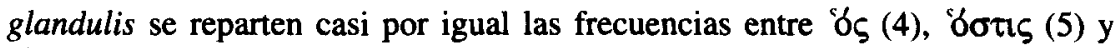
'ónov (4), mientras que De articulis presenta 15 veces 'o $\delta$ y el resto hasta 19 se

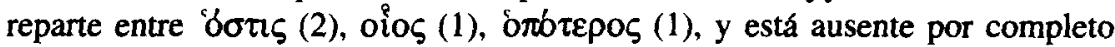
'ónov (para expresar el locativo «donde» prefiere la forma ñर, que he contado con el relativo). En este punto De articulis concuerda con la literatura del siglo $\mathrm{V}$ a. C., mientras que nuestro tratado refleja la progresión de la tendencia estadística que se inicia a finales de ese siglo y acaba casi por eliminar el simple ' $\delta \zeta^{12}$, al menos en nominativo. Lo que nos importa señalar aquí es que no antes del siglo IV a. C. el pronombre 'ó $\sigma \pi \zeta$ se ha convertido en un sinónimo del simple ' $\delta \zeta$, al que tiende a suplantar. En principio, pues, parece sugerente estudiar con mayor detalle el uso de las oraciones de relativo en este tratado. "Ootis aparece 5 veces $(2.2,3.1,6.1$ y dos veces en 16.1) introduciendo siempre relativas determinativas, como es lógico en este pronombre, pero en la mayor parte de los ejemplos nos encontramos con que el antecedente está claramente determinado y es, por lo tanto, identificable:

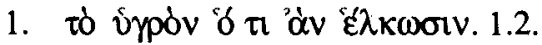

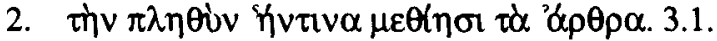

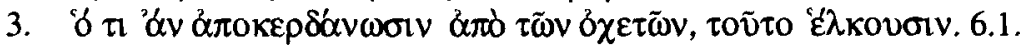

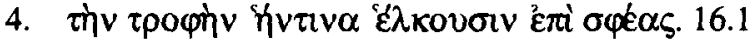

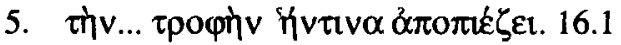

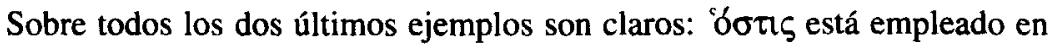
lugar del simple 'ós, el indicativo no deja lugar a dudas sobre este punto. Los ejemplos de 'óc son los siguientes:

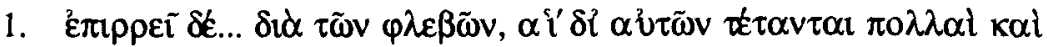
коÏ $\alpha \mathbf{l} .2 .2$.

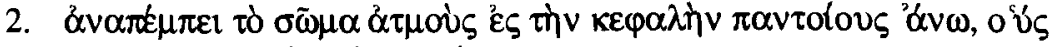

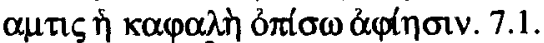

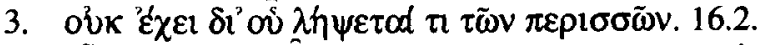

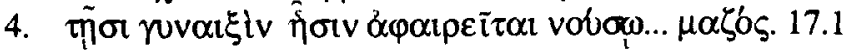

12 Cf. P. MONTEIL, La pharase relative en grec ancien, Paris 1963, pp. 124 ss., y 145, de las

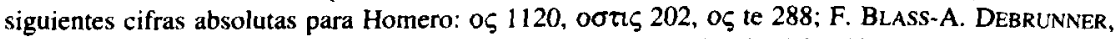
Grammatik des neutestamentlichen Griechisch, Göttingen, 1934, \$64.3, 293, en pp. 167 s.; A. N. JANNARIS, An historical Greek grammar, Oxford 1897, \& 1435; E. SCHWYZER-A. DEBRUNNER, Griechische Grammatik, München 1966, II p. 643. 
Aquí las oraciones de relativo se reparten por igual el uso determinativo, claro en los dos últimos ejemplos, y el uso disgresivo. En resumen, nos encontramos con un estado de lengua en el que el pronombre 'ó $\sigma \pi \zeta$ funciona como un sinónimo de insistencia con valor determinativo, pero ya ha perdido la noción de que su antecendente está indeterminado. El problema, pues, se limita a fijar en qué fecha ha ocurrido este fenómeno. Desgraciadamente P. Monteil no llega en su estudio más que hasta finales del siglo V. a C., cuando este fenómeno se empieza a manifestar, y lo único que nos dice es que en época del NT está completamente establecido. Este hecho apunta, pues, al siglo IV a. C., o a fecha posterior.

12. Esta fecha se puede contrastar mediante la comparación de la construcción de la frase en los autores del siglo IV a. C. y los del siglo II a. C., que forman el corpus estudiado por J. Blomqvist ${ }^{13}$; al menos el estudio de las desviaciones reducidas nos puede indicar a qué estilo se aproxima más nuestro tratado. Los resultados se recogen en los dos cuadros siguientes:

\section{Desviaciones reducidas, «z» entre S. IV ${ }^{14}$ y De glandulis}

\begin{tabular}{|c|c|c|c|c|c|c|}
\hline Part & s. $I V$ & $G l$ & Prob. & $\sigma x$ & $N x P$ & $z$ \\
\hline$\delta \dot{E}$ & 1059 & 55 & 0.3530 & 8.00 & 99.00 & -5.5 \\
\hline kod & 366 & 80 & 0.1220 & 5.48 & 34.00 & 8.39 \\
\hline$\alpha \lambda \lambda \alpha$ & 332 & 10 & 0.1107 & 5.25 & 31.00 & -4.0 \\
\hline$x \alpha \rho$ & 449 & 19 & 0.1497 & 5.97 & 42.00 & -3.8 \\
\hline asín. & 189 & 36 & 0.0630 & 4.07 & 18.00 & 4.42 \\
\hline orel & 54 & 15 & 0.0180 & 2.22 & 5.00 & 4.50 \\
\hline ocoor & 495 & 20 & 0.1650 & 6.21 & 46.00 & -4.1 \\
\hline osub & 56 & 45 & 0.0187 & 2.27 & 5.00 & 17.6 \\
\hline
\end{tabular}

orel $=" \delta \zeta$, 'ó $\sigma \pi \zeta$

Prob $=$ Probabilidad en el corpus del siglo IV.

$\sigma \mathrm{x}=$ Desviación tipo.

$\mathrm{N} \times \mathrm{P}=$ Cantidad teórica que sería de esperar en De glandulis.

$\mathrm{z}=$ Desviación reducida

13 Greek particles in Hellenistic prose, Lund 1969.

14 El corpus comprende 3.000 oraciones de Isócrates, Demóstenes y Aristóteles; los datos proceden de J. Blomqvist, o.c., p. $138 \mathrm{~s}$. 
Todas las desviaciones reducidas son significativas (el valor de $\mathrm{z}$ es siempre mayor que 3). Por lo tanto, se puede afirmar que este tratado no encaja en la situación linguística del siglo IV a. C., a pesar de la conclusión provisional a la que habíamos llegado.

Desviaciones entre autores del s. $\mathrm{II}^{15}$ y De glandulis

\begin{tabular}{|l|r|c|c|c|c|c|}
\hline Part & $s . I I$ & $G l$ & Prob. & $\sigma x$ & $N x P$ & $z$ \\
\hline$\delta \varepsilon$ & 870 & 55 & 0.4350 & 8.20 & 122.00 & -8.0 \\
$\kappa \alpha \mathfrak{\alpha}$ & 230 & 80 & 0.1150 & 5.34 & 32.00 & 8.99 \\
$\alpha \lambda \lambda \alpha \alpha$ & 176 & 10 & 0.0880 & 4.74 & 25.00 & -3.1 \\
$\gamma \alpha \rho$ & 278 & 19 & 0.1390 & 5.79 & 39.00 & -3.4 \\
asín. & 36 & 36 & 0.0180 & 2.22 & 5.00 & 13.9 \\
orel & 17 & 15 & 0.0085 & 1.54 & 2.00 & 8.44 \\
ocoor & 366 & 20 & 0.1830 & 6.47 & 51.00 & -4.7 \\
osub & 27 & 45 & 0.0135 & 1.93 & 4.00 & 21.2 \\
\hline
\end{tabular}

Las desviaciones son significativas también en todos los casos. Abundancia de $k \propto \alpha$, el asíndeton, oraciones de relativo y demás subordinadas, y escasez sobre todo de $\delta \notin$, son los rasgos que definen a De glandulis frente a la prosa del s. II a. C. Podemos concluir, por lo tanto, que la frecuencia de estas conectivas no corresponde con la esperada para un texto del siglo II.

13. Para contrastar el resultado hemos hecho los mismos cálculos con los todos autores del s. IV y del siglo II a. C., que emplea J. Blomqvist. Los resultados son los siguientes:

15 El corpus incluye 1.000 oraciones de Hiparco y otras tantas de Filodemo; los datos proceden igualmente de J. Blomqvist, l.c.. 


\section{Desviaciones reducidas con el total del s. IV}

\begin{tabular}{|c|c|c|c|c|c|c|}
\hline Part & s. $I V$ & $G l$ & Prob. & $\sigma x$ & $N x P$ & $z$ \\
\hline$\delta \dot{E}$ & 2220 & 55 & 0.4440 & 8.31 & 124.00 & -8.3 \\
\hline$\kappa \propto \alpha$ & 769 & 80 & 0.1538 & 6.04 & 43.00 & 6.13 \\
\hline$\alpha \lambda \lambda \alpha \alpha$ & 391 & 10 & 0.0782 & 4.49 & 22.00 & -2.6 \\
\hline$\gamma \alpha p$ & 575 & 19 & 0.1150 & 5.34 & 32.00 & -2.4 \\
\hline asín. & 241 & 36 & 0.0482 & 3.58 & 13.00 & 6.42 \\
\hline orel & 69 & 15 & 0.0138 & 1.95 & 4.00 & 5.64 \\
\hline ocoor & 673 & 20 & 0.1346 & 5.71 & 38.00 & -3.1 \\
\hline osub & 62 & 45 & 0.0124 & 1.85 & 3.00 & 22.7 \\
\hline
\end{tabular}

\section{Desviaciones reducidas con el total del s. II}

\begin{tabular}{|c|c|c|c|c|c|c|}
\hline Part & s. II & $G l$ & Prob. & $\sigma x$ & $N x P$ & $z$ \\
\hline$\delta \dot{\varepsilon}$ & 2070 & 55 & 0.5175 & 8.36 & 145.00 & -11 \\
\hline$x \alpha \mathfrak{l}$ & 421 & 80 & 0.1053 & 5.14 & 29.00 & 9.92 \\
\hline$\alpha \lambda \lambda \alpha x$ & 253 & 10 & 0.0633 & 4.07 & 18.00 & -1.9 \\
\hline$x \alpha p$ & 485 & 19 & 0.1213 & 5.46 & 34.00 & -2.7 \\
\hline asín. & 61 & 36 & 0.0153 & 2.05 & 4.00 & 15.6 \\
\hline orel & 87 & 15 & 0.0218 & 2.44 & 6.00 & 3.69 \\
\hline ocoor & 536 & 20 & 0.1340 & 5.70 & 38.00 & -3.1 \\
\hline osub & 87 & 45 & 0.0218 & 2.44 & 6.00 & 15.9 \\
\hline
\end{tabular}

Las diferencias siguen siendo significativas en la inmensa mayoría de las partículas salvo en los casos de $\alpha \lambda \lambda \alpha$ y de $\gamma \alpha \rho$, pero estos dos datos puede ser marginales, de forma que hemos de afirmar que no pertenece a la lengua de ninguna de estas dos centurias.

14. Esta conclusión nos plantea un problema. El tratado no puede ser anterior al siglo IV, según hemos visto que indican los usos sintácticos, y tampoco puede pertenecer a los siglos IV y II. Quedaría la posibilidad de que perteneciera al siglo III, por lo que hay que comprobar si hay una tendencia evolutiva coherente entre estos dos siglos que excluya, o haga difícil la datación de De 
glandulis en esa centuria. Con el fin de establecer esta tendencia hemos constituido dos corpora con los datos que nos ofrece J. Blomqvist; en el primero he agrupado todos los autores antiguos (Isócrates, Demóstenes, Aristóteles, Tucídides y Jenofonte), y en el segundo los recientes (Hiparco, Filodemo, Polibio y Diodoro de Sicilia), para someterlos a los mismos cálculos. Los resultados son los siguientes:

Desviaciones reducidas entre s. IV y s. II

\begin{tabular}{|l|c|c|c|c|c|c|}
\hline Part & $s . I V$ & $s . I I$ & Prob. & $\sigma x$ & $N x P$ & $z$ \\
\hline$\delta \xi$ & 2220 & 2070 & 0.4440 & 31.42 & 1776.0 & 9.36 \\
$\kappa \alpha \alpha$ & 769 & 421 & 0.1538 & 22.82 & 615.00 & -8.5 \\
$\alpha \lambda \lambda \alpha \alpha$ & 391 & 253 & 0.0782 & 16.98 & 313.00 & -3.5 \\
$\gamma \alpha \rho$ & 575 & 485 & 0.1150 & 20.18 & 460.00 & 1.24 \\
asín. & 241 & 61 & 0.0482 & 13.55 & 193.00 & -9.7 \\
orel & 69 & 87 & 0.0138 & 7.38 & 55.00 & 4.34 \\
ocoor & 673 & 536 & 0.1346 & 21.59 & 538.00 & -0.09 \\
osub & 62 & 87 & 0.0124 & 7.00 & 50.00 & 5.29 \\
\hline
\end{tabular}

Salvo en el caso de đáp y las demás partículas coordinadas las desviaciones son muy fuertes. Aumenta la frecuencia de las oraciones de relativo, las subordinadas ${ }^{16}$ y $\propto \xi$, disminuyen $\kappa \alpha \alpha, \alpha \lambda \lambda \alpha \alpha$ y el asíndeton. Comparando estos resultados con los obtenidos al contrastar De glandulis con la prosa del siglo IV (cf. $\S 12$ ), resulta que en cuatro casos, $\alpha \lambda \lambda \alpha$, orel, osub y ocoor, coincide con la prosa tardía y en otros cuatro el tratado muestra justamente la tendencia contraria ( $\delta \xi, \kappa \alpha \Upsilon, \gamma \alpha \propto p$, asín.). De todos estos datos parece deducirse que el tratado sigue la tendencia general de la lengua al aumentar las construcciones subordinadas e ir empobreciendo los medios de coordinación.

15. Hemos visto ya que las oraciones de relativo apuntan también hacia una fecha no anterior al siglo IV a. C. La situación que ofrecen las condicionales es la siguiente: el autor del tratado sólo conoce dos tipos, las introducidas

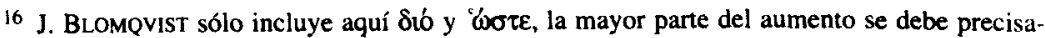


por ' $\eta \mathfrak{v}$ seguidas de subjuntivo y las introducidas por $\varepsilon l$, que siempre van acompañadas de un optativo, generalmente de aoristo. Los ejemplos son los siguientes:

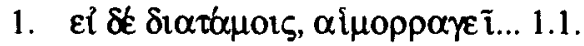

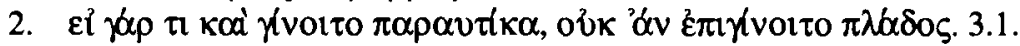

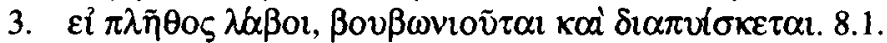

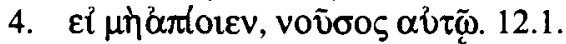

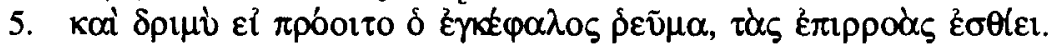
12.1 .

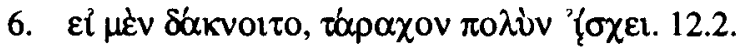

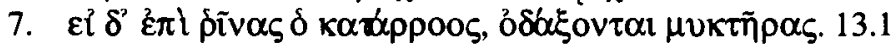

Salvo en el último ejemplo, en el que nos encontramos con una elipsis del verbo, el uso del optativo con el es constante, tanto cuando la principal está en presente de indicativo, como cuando aparece un irreal (2). Respecto a los ejemplos introducidos por " $\mathrm{h} v$ se puede decir que en la principal suele aparecer un presente de valor genérico ${ }^{17}$ en combinación con un subjuntivo en la prótasis o cualquier tiempo del indicativo. Pero la combinación de un presente también genérico con una prótasis en optativo es algo menos frecuente. De hecho los ejemplos de este esquema que recoge $\mathrm{W}$. Goodwin ${ }^{18}$ pertenecen en su mayor parte a la lengua poética (Píndaro, Homero, Hesíodo, Sófocles), aunque tenemos un primer ejemplo, discutido, en la prosa de Tucídides (2. 39) y otro en Demóstenes (18.21). El hecho de que ambas construcciones alternen libremente en la misma frase invita a pensar en los ejemplos recogidos por Schwyzer ${ }^{19}$ en los que aparecen una al lado de otra los dos tipos de prótasis: Homero, Heródoto, Platón, Eurípides, Jenofonte y Demóstenes. Pero a pesar de estos paralelos, hay que tener en cuenta que la combinación de una prótasis en optativo con un presente general en la apódosis es propia de la lengua poética y de tono elevado.

16. Por sí sólo el estilo del tratado, la frecuencia del asíndeton y el tono sentencioso de sus frases, hablan de las pretensiones literarias de su autor. Al respecto no está de más recordar los abundantes términos poéticos que salpican

mente a $\delta$.

17 Cf. A. Ruksbaron, The syntax and semantic of the verb in Classical Greek, Amsterdam 1984 , p. 71. Los ejemplos aparecen en los siguientes lugares: $1.1,2.2,7.2,7.3,12.1,12.2,14.1$, 14.2 .

18 Syntax of the moods and tenses of the Greek verb, London $1897, \$ \$ 500,501$. 
su prosa, algunos de ellos estudiados ya por H. Mørland (l.c.) y algunos términos técnicos que no son muy frecuentes en el $\mathrm{CH}^{20}$. A esos poetismos podemos añadir la construcción con optativo de las condicionales. Estos datos por sí solos son indicios de una fecha reciente para el tratado. La relativa pobreza de las partículas de coordinación ( $(9)$, la confusión del uso de los pronombres relativos $(\S 11)$, las rarezas como $\delta$ y y cot $(\S 5$, nota 7$)$, son otros datos que corroboran esta primera impresión. En cualquier caso, podemos afirmar que el tratado no puede ser antiguo, según demuestran las estadísticas, y corroboran los hechos linguísticos. Por lo tanto, se impone la obligación de intentar encontrar una fecha posterior para el tratado. Con ese fin he comparado los datos que venimos comentando con la frecuencia de las partículas en el Nuevo Testamento $^{21}$ :

Desviaciones reducidas entre NT y De gland

\begin{tabular}{|c|c|c|c|c|c|c|}
\hline Part & $N T$ & $G l$ & Prob. & $\sigma x$ & $N \times P$ & $z$ \\
\hline$\delta \varepsilon$ & 2771 & 55 & 0.1364 & 6.36 & 47.00 & 1.26 \\
\hline$k \alpha \alpha$ & 8947 & 169 & 0.4403 & 9.19 & 151.00 & 1.96 \\
\hline$\not \alpha \rho \rho$ & 1036 & 19 & 0.0510 & 4.07 & 17.00 & 0.49 \\
\hline$\alpha \lambda \lambda \alpha$ & 635 & 10 & 0.0313 & 3.22 & 11.00 & -0.31 \\
\hline asín. & 856 & 23 & 0.0421 & 3.72 & 14.00 & 2.42 \\
\hline orel & 1633 & 15 & 0.0804 & 5.04 & 28.00 & -2.58 \\
\hline ocoor & 1600 & 30 & 0.0787 & 4.99 & 27.00 & 0.60 \\
\hline osub & 2840 & 22 & 0.1398 & 6.42 & 48.00 & -4.05 \\
\hline
\end{tabular}

Las desviaciones sólo son significativas en la frecuencia de las oraciones de relativo y en los demás medios de subordinación. En cualquier caso este resultado sólo tiene un significado negativo. Es decir, no se puede determinar que ambos corpora sean distintos, lo que no quiere decir que sean iguales o

\footnotetext{
Ty E. SCHWyzer- A. Debrunner, Griechische Grammatik, München 1966, II p. 686 s.

20 Algunos de estos términos se estudian en nuestro "Las fuentes del tratado De glandulis», publicado en J.A. LOPEZ FEREZ (ed.), Tratados hipocríticos (estudios acerca de su contenido, forma $e$ influencia). Actas del VII Colloque International hippocratique (Madrid, 24-29 de septiembre de 1990), Madrid 1992, pp. 421-435.
} 
pertenezcan al mismo autor o época. Una demostración de ese tipo no se puede obtener directamente por procedimientos estadísticos, pero al menos gana verosimilitud la hipótesis de que este tratado es una imitación de época alejandrina o incluso posterior, que era la tesis defendida por Diels, Mørland y Galeno (XVIII A Kühn), ya que los datos estadísticos excluyen una datación en los siglos IV y II a. C. El valor que aproxima la distribución de las partículas en $D e$ glandulis y De articulis $(\S 8)$ es un efecto de la imitación intencionada del estilo.

21 Datos tomados de R. MORGENTHALER, Statistik de Neutestamentlichen Wortschatzes, Zuirich-Stuttgart, $1973^{2}$. 